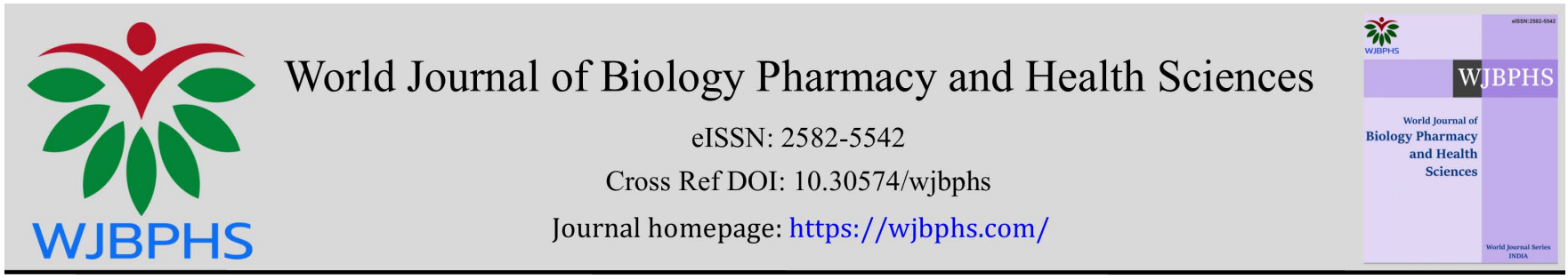

(RESEARCH ARTiCLE)

\title{
Pollutants and climatic conditions related to the childhood asthma and atopic dermatitis
}

\author{
Shigeru Suna * \\ Private Health Research Laboratory, 14-22 Shinkita-machi, Takamatsu-shi, Kagawa 760-0001, Japan.
}

World Journal of Biology Pharmacy and Health Sciences, 2022, 09(01), 027-038

Publication history: Received on 12 December 2021; revised on 19 January 2022; accepted on 21 January 2022

Article DOI: https://doi.org/10.30574/wjbphs.2022.9.1.0025

\begin{abstract}
Indoor, outdoor pollutants and climatic conditions in the growing environment can develop childhood asthma and atopic dermatitis. Therefore, it is important to identify the environmental burden of the community on the risk of childhood asthma and atopic dermatitis.

To clarify the relationship between regional prevalence rate of childhood asthma and atopic dermatitis among the first grade elementary school students and preschool indoor and outdoor conditions such as smoking rate, photochemical oxidants concentration, ambient temperature and relative humidity, multiple linear regression analysis was performed.

Stepwise multiple regression analysis with asthma rate as the objective variable, atopic dermatitis rate, smoking rate, photochemical oxidants, ambient temperature and relative humidity as explanatory variables, revealed that atopic dermatitis rate and smoking rate were significant independent variables. This result suggests that tobacco smoke is a risk factor for non-atopic asthma rather than atopic asthma.

Stepwise multiple regression analysis with atopic dermatitis rate among first grade elementary school students as the objective variable, smoking rate, photochemical oxidants, ambient temperature and relative humidity as explanatory variables, revealed that photochemical oxidants and ambient temperature were significant independent variable.

Present study suggests that preschool indoor and outdoor conditions such as environmental tobacco smoke, photochemical oxidants, and ambient temperature may be associated with the development of childhood asthma and atopic dermatitis.
\end{abstract}

Keywords: Childhood asthma; Atopic dermatitis; Smoking rate; Photochemical oxidants; Ambient temperature; Relative humidity

\section{Introduction}

Indoor, outdoor pollutants and climatic conditions in the growing environment can develop childhood asthma and atopic dermatitis [1-7]. Therefore, it is important to identify the environmental burden of the community on the risk of childhood asthma and atopic dermatitis.

The aim of this study is to clarify the relationship between the regional prevalence rate of childhood asthma and atopic dermatitis among the first grade of elementary school students in Japan and the preschool environmental conditions such as smoking rate, photochemical oxidants concentration, ambient temperature and relative humidity.

\footnotetext{
* Corresponding author: Shigeru Suna

Private Health Research Laboratory, 14-22 Shinkita-machi, Takamatsu-shi, Kagawa 760-0001, Japan. 


\section{Material and methods}

\subsection{Prevalence rate of childhood asthma and atopic dermatitis}

Prefectural prevalence rate of childhood asthma and atopic dermatitis among the first grade of elementary school were from School Health Statistics Survey by the Ministry of Education, Culture, Sports, Science and Technology (https://www.e-stat.go.jp/stat-search/files?page=1\&toukei=00400002\&tstat=000001011648).

\subsection{Air pollutants}

Smoking rate by prefecture, as an indicator of regional indoor air pollution was obtained from Cancer Information Service, National Cancer Center, Japan.

(https://ganjoho.jp/reg_stat/statistics/dl/index.html\#smoking). The smoking rate is the ratio of the total number of people (men and women over 20 years old) who answered "smoking daily" or "sometimes smoked" as a numerator and the "total number of respondents" as a denominator.

The annual average value of prefectural photochemical oxidants concentration was obtained from National Institute for Environmental Studies.

(https://tenbou.nies.go.jp/gis/monitor/?map_mode=jpn_env_atmosphere)

\subsection{Climatic conditions}

The annual average values of relative humidity and ambient temperature by prefecture were from Social Indicators by Prefecture. The values were downloaded from e-Stat (https://www.e-stat.go.jp/dbview?sid=0000010102).

\subsection{Statistical analysis}

Stepwise multiple linear regression analysis was performed to determine the relationship between prevalence rate of childhood asthma and atopic dermatitis and preschool environmental conditions. $\mathrm{p}<0.05$ was considered as statistically significant. The first investigation was for first grade elementary school students from 2008 to 2010, and the second was for first grade elementary school students from 2017 to 2019.

\section{Results}

\subsection{Prevalence rate of childhood asthma and atopic dermatitis}

Table 1and 2 show percentage of asthma among first grade elementary school students from 2008 to 2010 and from 2017 to 2019. Table 3 and Table 4 shows percentage of atopic dermatitis among first grade elementary school students from 2008 to 2010 and from 2017 to 2019.

Table 1 Prevalence rate of asthma among first grade elementary school students from 2008 to 2010

\begin{tabular}{|l|c|c|c|c|c|c|c|}
\hline \multirow{2}{*}{ Prefecture } & \multicolumn{3}{|c|}{ Asthma (\%) } & \multirow{2}{*}{ Prefecture } & \multicolumn{3}{c|}{ Asthma (\%) } \\
\cline { 2 - 4 } & 2008 & 2009 & 2010 & & 2008 & 2009 & 2010 \\
\hline Hokkaido & 2.6 & 4.1 & 2.5 & Shiga & 2.9 & 1.7 & 3.2 \\
\hline Aomori & 2.6 & 2.5 & 2.1 & Kyoto & 3.6 & 3.3 & 4.5 \\
\hline Iwate & 3.8 & 2.0 & 3.9 & Osaka & 3.4 & 4.4 & 3.9 \\
\hline Miyagi & 6.4 & 5.9 & 6.8 & Hyogo & 3.8 & 2.7 & 3.0 \\
\hline Akita & 5.3 & 4.1 & 6.5 & Nara & 1.1 & 1.6 & 1.2 \\
\hline Yamagata & 4.5 & 3.5 & 6.3 & Wakayama & 1.1 & 1.5 & 1.7 \\
\hline Fukushima & 3.5 & 3.3 & 3.1 & Tottori & 7.5 & 7.9 & 8.3 \\
\hline Ibaraki & 4.8 & 4.1 & 7.3 & Shimane & 4.3 & 5.6 & 6.3 \\
\hline
\end{tabular}


World Journal of Biology Pharmacy and Health Sciences, 2022, 09(01), 027-038

\begin{tabular}{|l|c|c|c|c|c|c|c|}
\hline Tochigi & 3.0 & 4.1 & 5.1 & Okayama & 3.9 & 3.5 & 6.5 \\
\hline Gunma & 5.1 & 4.7 & 4.2 & Hiroshima & 3.4 & 4.1 & 3.8 \\
\hline Saitama & 4.1 & 3.9 & 6.0 & Yamaguchi & 3.2 & 3.3 & 5.1 \\
\hline Chiba & 5.7 & 4.8 & 5.6 & Tokushima & 4.0 & 4.1 & 3.8 \\
\hline Tokyo & 6.6 & 7.0 & 6.6 & Kagawa & 4.4 & 2.1 & 4.5 \\
\hline Kanagawa & 5.4 & 3.5 & 5.9 & Ehime & 3.1 & 2.9 & 2.9 \\
\hline Niigata & 7.5 & 6.2 & 7.9 & Kochi & 3.0 & 2.9 & 3.3 \\
\hline Toyama & 3.8 & 2.3 & 5.9 & Fukuoka & 2.7 & 2.0 & 4.2 \\
\hline Ishikawa & 2.0 & 2.1 & 3.1 & Saga & 1.9 & 1.2 & 3.5 \\
\hline Fukui & 5.3 & 3.2 & 5.1 & Nagasaki & 2.7 & 2.0 & 2.7 \\
\hline Yamanashi & 4.4 & 3.3 & 4.5 & Kumamoto & 2.8 & 1.8 & 2.8 \\
\hline Nagano & 4.8 & 4.7 & 7.3 & Oita & 1.1 & 1.9 & 2.7 \\
\hline Gifu & 2.9 & 2.1 & 2.4 & Miyazaki & 3.2 & 2.2 & 3.3 \\
\hline Shizuoka & 5.0 & 3.5 & 3.6 & Kagoshima & 2.5 & 1.7 & 2.3 \\
\hline Aichi & 5.2 & 4.1 & 5.3 & Okinawa & 2.2 & 2.1 & 3.4 \\
\hline Mie & 3.2 & 2.4 & 4.0 & mean (SD) & $3.9(1.6)$ \\
\hline
\end{tabular}

Table 2 Prevalence rate of asthma among first grade elementary school students from 2017 to 2019

\begin{tabular}{|l|c|c|c|l|c|c|c|}
\hline \multirow{2}{*}{ Prefecture } & \multicolumn{3}{|c|}{ Asthma (\%) } & \multirow{2}{*}{ Prefecture } & \multicolumn{3}{|c|}{ Asthma (\%) } \\
\cline { 2 - 4 } \cline { 7 - 8 } & 2017 & 2018 & 2019 & & 2017 & 2018 & 2019 \\
\hline Hokkaido & 5.8 & 3.9 & 4.0 & Shiga & 3.3 & 2.2 & 2.0 \\
\hline Aomori & 1.5 & 1.2 & 1.8 & Kyoto & 4.0 & 3.5 & 2.6 \\
\hline Iwate & 5.3 & 3.0 & 3.8 & Osaka & 2.6 & 2.5 & 2.4 \\
\hline Miyagi & 6.2 & 5.4 & 5.1 & Hyogo & 4.1 & 3.5 & 3.2 \\
\hline Akita & 4.5 & 3.2 & 3.3 & Nara & 1.1 & 1.0 & 2.0 \\
\hline Yamagata & 5.9 & 4.6 & 4.6 & Wakayama & 1.6 & 2.6 & 1.8 \\
\hline Fukushima & 3.6 & 3.9 & 1.4 & Tottori & 6.1 & 5.4 & 5.3 \\
\hline Ibaraki & 6.5 & 5.3 & 6.2 & Shimane & 4.5 & 3.5 & 6.1 \\
\hline Tochigi & 6.3 & 4.6 & 4.7 & Okayama & 3.7 & 3.6 & 5.3 \\
\hline Gunma & 3.5 & 4.3 & 4.5 & Hiroshima & 2.5 & 2.6 & 2.4 \\
\hline Saitama & 3.7 & 2.3 & 3.3 & Yamaguchi & 3.1 & 4.6 & 2.1 \\
\hline Chiba & 5.3 & 6.6 & 5.6 & Tokushima & 3.0 & 3.4 & 2.6 \\
\hline Tokyo & 4.3 & 4.3 & 3.7 & Kagawa & 4.0 & 3.9 & 2.7 \\
\hline Kanagawa & 5.8 & 4.7 & 5.3 & Ehime & 2.7 & 2.8 & 3.0 \\
\hline Niigata & 7.8 & 6.2 & 6.7 & Kochi & 2.8 & 4.2 & 1.4 \\
\hline Toyama & 3.9 & 4.8 & 4.1 & Fukuoka & 4.2 & 2.0 & 1.9 \\
\hline Ishikawa & 1.9 & 1.9 & 2.6 & Saga & 2.7 & 4.2 & 2.9 \\
\hline
\end{tabular}


World Journal of Biology Pharmacy and Health Sciences, 2022, 09(01), 027-038

\begin{tabular}{|l|l|l|l|l|l|l|l|}
\hline Fukui & 2.2 & 2.9 & 2.2 & Nagasaki & 3.4 & 3.2 & 4.0 \\
\hline Yamanashi & 3.6 & 3.9 & 2.5 & Kumamoto & 3.1 & 1.2 & 1.2 \\
\hline Nagano & 5.2 & 5.8 & 4.9 & Oita & 2.7 & 2.5 & 3.4 \\
\hline Gifu & 1.7 & 2.6 & 2.0 & Miyazaki & 3.5 & 3.0 & 3.4 \\
\hline Shizuoka & 3.1 & 3.1 & 2.3 & Kagoshima & 2.7 & 2.1 & 3.1 \\
\hline Aichi & 3.5 & 4.3 & 4.3 & Okinawa & 2.5 & 2.2 & 1.7 \\
\hline Mie & 3.3 & 3.1 & 2.7 & mean (SD) & \multicolumn{3}{|c|}{$3.5(1.4)$} \\
\hline
\end{tabular}

Table3 Prevalence rate of atopic dermatitis among first grade elementary school students from 2008 to 2010

\begin{tabular}{|l|c|c|c|l|c|c|c|}
\hline \multirow{2}{*}{ Prefecture } & \multicolumn{3}{|c|}{ Atopic dermatitis (\%) } & Prefecture & \multicolumn{3}{|c|}{ Atopic dermatitis (\%) } \\
\cline { 2 - 4 } & 2008 & 2009 & 2010 & & 2008 & 2009 & 2010 \\
\hline Hokkaido & 2.4 & 3.7 & 1.8 & Shiga & 4.3 & 2.7 & 3.3 \\
\hline Aomori & 1.5 & 1.5 & 1.6 & Kyoto & 4.6 & 4.6 & 4.9 \\
\hline Iwate & 3.7 & 2.8 & 2.2 & Osaka & 2.8 & 2.9 & 2.6 \\
\hline Miyagi & 4.9 & 5.6 & 4.8 & Hyogo & 3.9 & 3.4 & 3.0 \\
\hline Akita & 5.3 & 4.1 & 3.7 & Nara & 2.1 & 2.2 & 3.3 \\
\hline Yamagata & 4.1 & 4.0 & 2.8 & Wakayama & 3.3 & 2.2 & 2.2 \\
\hline Fukushima & 2.9 & 3.6 & 2.9 & Tottori & 8.1 & 7.1 & 7.6 \\
\hline Ibaraki & 3.9 & 3.7 & 5.5 & Shimane & 5.9 & 6.3 & 5.6 \\
\hline Tochigi & 3.3 & 2.1 & 2.7 & Okayama & 2.9 & 2.5 & 4.0 \\
\hline Gunma & 3.2 & 3.0 & 2.3 & Hiroshima & 2.6 & 3.5 & 3.4 \\
\hline Saitama & 3.2 & 2.6 & 3.5 & Yamaguchi & 3.1 & 2.1 & 2.8 \\
\hline Chiba & 2.8 & 2.6 & 2.9 & Tokushima & 3.8 & 3.9 & 5.0 \\
\hline Tokyo & 6.0 & 4.2 & 4.8 & Kagawa & 4.1 & 2.9 & 4.3 \\
\hline Kanagawa & 2.4 & 2.0 & 3.1 & Ehime & 2.0 & 1.8 & 1.7 \\
\hline Niigata & 6.2 & 5.5 & 4.4 & Kochi & 2.9 & 2.8 & 2.5 \\
\hline Toyama & 4.5 & 4.0 & 4.0 & Fukuoka & 3.4 & 1.8 & 3.5 \\
\hline Ishikawa & 3.0 & 4.1 & 3.6 & Saga & 2.8 & 2.4 & 2.9 \\
\hline Fukui & 7.4 & 8.5 & 7.3 & Nagasaki & 3.4 & 3.0 & 5.1 \\
\hline Yamanashi & 4.4 & 2.5 & 2.9 & Kumamoto & 1.9 & 1.3 & 2.4 \\
\hline Nagano & 4.4 & 4.5 & 5.5 & Oita & 1.8 & 2.3 & 1.9 \\
\hline Gifu & 4.0 & 3.3 & 4.5 & Miyazaki & 3.1 & 1.8 & 2.2 \\
\hline Shizuoka & 4.1 & 3.8 & 3.1 & Kagoshima & 1.9 & 0.9 & 1.9 \\
\hline Aichi & 6.2 & 4.0 & 5.0 & Okinawa & 2.1 & 2.3 & 2.8 \\
\hline Mie & 4.0 & 2.8 & 3.3 & mean (SD) & & $3.5(1.4)$ \\
\hline
\end{tabular}


Table 4 Prevalence rate of atopic dermatitis among first grade elementary school students from 2017 to 2019

\begin{tabular}{|l|c|c|c|l|c|c|c|}
\hline \multirow{2}{*}{ Prefecture } & \multicolumn{3}{|c|}{ Atopic dermatitis (\%) } & Prefecture & \multicolumn{3}{|c|}{ Atopic dermatitis (\%) } \\
\cline { 2 - 4 } & $\mathbf{2 0 1 7}$ & $\mathbf{2 0 1 8}$ & $\mathbf{2 0 1 9}$ & & $\mathbf{2 0 1 7}$ & $\mathbf{2 0 1 8}$ & $\mathbf{2 0 1 9}$ \\
\hline Hokkaido & 4.8 & 4.0 & 4.5 & Shiga & 3.2 & 2.8 & 3.0 \\
\hline Aomori & 1.6 & 1.5 & 1.0 & Kyoto & 5.0 & 5.0 & 3.5 \\
\hline Iwate & 2.5 & 3.9 & 3.7 & Osaka & 1.7 & 3.0 & 2.7 \\
\hline Miyagi & 4.3 & 5.4 & 3.3 & Hyogo & 3.4 & 3.0 & 2.5 \\
\hline Akita & 5.2 & 3.9 & 3.8 & Nara & 3.4 & 1.9 & 3.9 \\
\hline Yamagata & 4.2 & 3.4 & 3.0 & Wakayama & 2.9 & 2.9 & 2.8 \\
\hline Fukushima & 2.4 & 2.9 & 1.3 & Tottori & 5.9 & 6.0 & 5.7 \\
\hline Ibaraki & 6.9 & 5.4 & 4.6 & Shimane & 4.6 & 5.5 & 6.3 \\
\hline Tochigi & 3.1 & 4.3 & 4.0 & Okayama & 2.9 & 4.1 & 4.1 \\
\hline Gunma & 2.0 & 2.7 & 3.6 & Hiroshima & 3.0 & 2.7 & 2.5 \\
\hline Saitama & 3.3 & 2.8 & 2.3 & Yamaguchi & 2.2 & 3.8 & 1.4 \\
\hline Chiba & 3.0 & 3.6 & 3.8 & Tokushima & 2.2 & 3.0 & 3.4 \\
\hline Tokyo & 3.9 & 3.7 & 3.9 & Kagawa & 2.4 & 2.7 & 3.1 \\
\hline Kanagawa & 2.5 & 3.1 & 2.8 & Ehime & 2.7 & 2.0 & 2.7 \\
\hline Niigata & 4.9 & 4.7 & 6.3 & Kochi & 2.7 & 2.7 & 2.4 \\
\hline Toyama & 2.6 & 2.7 & 2.9 & Fukuoka & 2.8 & 1.7 & 1.5 \\
\hline Ishikawa & 2.2 & 2.8 & 2.3 & Saga & 2.7 & 2.6 & 3.1 \\
\hline Fukui & 5.7 & 4.3 & 4.0 & Nagasaki & 3.6 & 3.3 & 4.5 \\
\hline Yamanashi & 3.6 & 2.5 & 2.4 & Kumamoto & 1.7 & 2.1 & 2.2 \\
\hline Nagano & 3.0 & 5.1 & 3.9 & Oita & 1.5 & 1.1 & 1.7 \\
\hline Gifu & 3.4 & 3.5 & 3.8 & Miyazaki & 1.6 & 1.5 & 1.6 \\
\hline Shizuoka & 2.5 & 4.3 & 2.3 & Kagoshima & 1.9 & 1.7 & 2.2 \\
\hline Aichi & 4.9 & 6.7 & 6.3 & Okinawa & 1.6 & 2.6 & 1.7 \\
\hline Mie & 2.6 & 2.9 & 2.7 & mean (SD) & & $3.3(1.3)$ \\
\hline
\end{tabular}

\subsection{Air pollutants and climatic conditions in the preschool environment}

Table 5 and 6 show average values of photochemical oxidants in the preschool environment. Table 7 and 8 show smoking rate, ambient temperature and relative humidity. 
Table 5 Average concentration of photochemical oxidants in the preschool environment for first grade elementary school students from 2007 to 2019

\begin{tabular}{|c|c|c|c|c|c|c|c|}
\hline \multirow[t]{2}{*}{ Prefecture } & \multicolumn{3}{|c|}{$\begin{array}{c}\text { Average concentration of } \\
\text { photochemical oxidants (ppm) }\end{array}$} & \multirow[t]{2}{*}{ Prefecture } & \multicolumn{3}{|c|}{$\begin{array}{c}\text { Average concentration of } \\
\text { photochemical oxidants (ppm) }\end{array}$} \\
\hline & $\begin{array}{l}2003- \\
2007\end{array}$ & $\begin{array}{l}2004- \\
2008 \\
\end{array}$ & $\begin{array}{l}2005- \\
2009\end{array}$ & & $\begin{array}{l}2003- \\
2007 \\
\end{array}$ & $\begin{array}{l}2004- \\
2008\end{array}$ & $\begin{array}{l}2005- \\
2009\end{array}$ \\
\hline Hokkaido & 0.028 & 0.029 & 0.029 & Shiga & 0.034 & 0.035 & 0.035 \\
\hline Aomori & 0.035 & 0.035 & 0.034 & Kyoto & 0.031 & 0.032 & 0.033 \\
\hline Iwate & 0.028 & 0.029 & 0.029 & Osaka & 0.031 & 0.031 & 0.031 \\
\hline Miyagi & 0.030 & 0.030 & 0.031 & Hyogo & 0.031 & 0.032 & 0.032 \\
\hline Akita & 0.037 & 0.037 & 0.037 & Nara & 0.031 & 0.031 & 0.031 \\
\hline Yamagata & 0.034 & 0.034 & 0.033 & Wakayama & 0.032 & 0.033 & 0.033 \\
\hline Fukushima & 0.031 & 0.031 & 0.032 & Tottori & 0.033 & 0.033 & 0.034 \\
\hline Ibaraki & 0.032 & 0.032 & 0.032 & Shimane & 0.038 & 0.038 & 0.038 \\
\hline Tochigi & 0.029 & 0.030 & 0.031 & Okayama & 0.029 & 0.030 & 0.031 \\
\hline Gunma & 0.032 & 0.032 & 0.033 & Hiroshima & 0.033 & 0.033 & 0.034 \\
\hline Saitama & 0.029 & 0.030 & 0.030 & Yamaguchi & 0.031 & 0.032 & 0.033 \\
\hline Chiba & 0.031 & 0.031 & 0.030 & Tokushima & 0.036 & 0.036 & 0.036 \\
\hline Tokyo & 0.028 & 0.028 & 0.029 & Kagawa & 0.029 & 0.029 & 0.030 \\
\hline Kanagawa & 0.026 & 0.027 & 0.027 & Ehime & 0.029 & 0.029 & 0.030 \\
\hline Niigata & 0.033 & 0.034 & 0.035 & Kochi & 0.029 & 0.030 & 0.030 \\
\hline Toyama & 0.036 & 0.036 & 0.036 & Fukuoka & 0.031 & 0.031 & 0.031 \\
\hline Ishikawa & 0.035 & 0.036 & 0.036 & Saga & 0.031 & 0.032 & 0.033 \\
\hline Fukui & 0.031 & 0.031 & 0.032 & Nagasaki & 0.033 & 0.034 & 0.035 \\
\hline Yamanashi & 0.032 & 0.032 & 0.032 & Kumamoto & 0.028 & 0.029 & 0.030 \\
\hline Nagano & 0.031 & 0.032 & 0.032 & Oita & 0.028 & 0.028 & 0.029 \\
\hline Gifu & 0.029 & 0.031 & 0.032 & Miyazaki & 0.032 & 0.032 & 0.032 \\
\hline Shizuoka & 0.030 & 0.031 & 0.032 & Kagoshima & 0.034 & 0.034 & 0.034 \\
\hline Aichi & 0.028 & 0.029 & 0.030 & Okinawa & 0.026 & 0.026 & 0.027 \\
\hline Mie & 0.031 & 0.032 & 0.032 & mean (SD) & & $32(0,0$ & \\
\hline
\end{tabular}

Table 6 Average concentration of photochemical oxidants in the pre-school environment for first grade elementary school students from 2017 to 2019

\begin{tabular}{|c|c|c|c|c|c|c|c|}
\hline \multirow[t]{2}{*}{ Prefecture } & \multicolumn{3}{|c|}{$\begin{array}{c}\text { Average concentration of } \\
\text { photochemical oxidants (ppm) }\end{array}$} & \multirow[t]{2}{*}{ Prefecture } & \multicolumn{3}{|c|}{$\begin{array}{c}\text { Average concentration of } \\
\text { photochemical oxidants (ppm) }\end{array}$} \\
\hline & 2012-2016 & 2013-2017 & 2014-2018 & & 2012-2016 & 2013-2017 & 2014-2018 \\
\hline Hokkaido & 0.031 & 0.031 & 0.031 & Shiga & 0.036 & 0.036 & 0.036 \\
\hline Aomori & 0.033 & 0.033 & 0.034 & Kyoto & 0.034 & 0.034 & 0.034 \\
\hline Iwate & 0.031 & 0.031 & 0.032 & Osaka & 0.032 & 0.032 & 0.033 \\
\hline Miyagi & 0.032 & 0.032 & 0.033 & Hyogo & 0.032 & 0.032 & 0.033 \\
\hline Akita & 0.036 & 0.036 & 0.036 & Nara & 0.032 & 0.032 & 0.033 \\
\hline Yamagata & 0.035 & 0.035 & 0.035 & Wakayama & 0.036 & 0.036 & 0.036 \\
\hline Fukushima & 0.033 & 0.033 & 0.034 & Tottori & 0.036 & 0.036 & 0.036 \\
\hline
\end{tabular}


World Journal of Biology Pharmacy and Health Sciences, 2022, 09(01), 027-038

\begin{tabular}{|l|l|l|l|l|l|l|l|}
\hline Ibaraki & 0.033 & 0.033 & 0.033 & Shimane & 0.039 & 0.039 & 0.040 \\
\hline Tochigi & 0.032 & 0.032 & 0.033 & Okayama & 0.032 & 0.032 & 0.032 \\
\hline Gunma & 0.037 & 0.037 & 0.037 & Hiroshima & 0.033 & 0.033 & 0.033 \\
\hline Saitama & 0.033 & 0.033 & 0.033 & Yamaguchi & 0.035 & 0.035 & 0.035 \\
\hline Chiba & 0.032 & 0.032 & 0.033 & Tokushima & 0.035 & 0.035 & 0.036 \\
\hline Tokyo & 0.031 & 0.031 & 0.031 & Kagawa & 0.033 & 0.033 & 0.034 \\
\hline Kanagawa & 0.031 & 0.031 & 0.031 & Ehime & 0.032 & 0.032 & 0.033 \\
\hline Niigata & 0.037 & 0.037 & 0.037 & Kochi & 0.034 & 0.034 & 0.034 \\
\hline Toyama & 0.037 & 0.037 & 0.037 & Fukuoka & 0.033 & 0.033 & 0.034 \\
\hline Ishikawa & 0.038 & 0.038 & 0.038 & Saga & 0.036 & 0.036 & 0.037 \\
\hline Fukui & 0.037 & 0.037 & 0.037 & Nagasaki & 0.036 & 0.036 & 0.036 \\
\hline Yamanashi & 0.033 & 0.033 & 0.033 & Kumamoto & 0.034 & 0.034 & 0.034 \\
\hline Nagano & 0.034 & 0.034 & 0.035 & Oita & 0.032 & 0.032 & 0.033 \\
\hline Gifu & 0.034 & 0.034 & 0.034 & Miyazaki & 0.029 & 0.029 & 0.029 \\
\hline Shizuoka & 0.034 & 0.034 & 0.034 & Kagoshima & 0.031 & 0.031 & 0.031 \\
\hline Aichi & 0.032 & 0.032 & 0.032 & Okinawa & 0.030 & 0.031 & 0.030 \\
\hline Mie & 0.035 & 0.035 & 0.035 & mean (SD) & & $0.034(0.002)$ & \\
\hline
\end{tabular}

Table 7 Smoking rate and climatic conditions in the pre-school environment for first grade elementary school students from 2008 to 2010

\begin{tabular}{|c|c|c|c|c|c|c|c|}
\hline Prefecture & $\begin{array}{l}{ }^{\#} \text { Smoking } \\
\text { rate (\%) }\end{array}$ & $\begin{array}{c}{ }^{\# \#} \text { Ambient } \\
\text { temperature } \\
\text { (?) }\end{array}$ & $\begin{array}{c}\text { \#\#\# Relative } \\
\text { humidity } \\
\text { (\%) }\end{array}$ & Prefecture & $\begin{array}{l}{ }^{\#} \text { Smoking } \\
\text { rate (\%) }\end{array}$ & $\begin{array}{c}{ }^{\# \#} \text { Ambient } \\
\text { temperature } \\
\text { (囵) }\end{array}$ & $\begin{array}{c}\text { \#\#\# Relative } \\
\text { humidity } \\
\text { (\%) }\end{array}$ \\
\hline Hokkaido & 30.5 & 9.3 & 68 & Shiga & 23.8 & 15.1 & 74 \\
\hline Aomori & 28.5 & 10.7 & 75 & Kyoto & 23.3 & 16.2 & 63 \\
\hline Iwate & 25.0 & 10.5 & 72 & Osaka & 25.9 & 17.4 & 63 \\
\hline Miyagi & 26.8 & 12.7 & 72 & Hyogo & 22.9 & 17.2 & 65 \\
\hline Akita & 25.3 & 12.1 & 73 & Nara & 21.4 & 15.2 & 71 \\
\hline Yamagata & 24.3 & 12.0 & 75 & Wakayama & 23.3 & 17.1 & 62 \\
\hline Fukushima & 26.5 & 13.3 & 69 & Tottori & 22.2 & 15.3 & 72 \\
\hline Ibaraki & 25.7 & 14.0 & 73 & Shimane & 20.9 & 15.4 & 73 \\
\hline Tochigi & 26.5 & 14.4 & 69 & Okayama & 23.4 & 16.7 & 66 \\
\hline Gunma & 26.4 & 15.0 & 61 & Hiroshima & 23.2 & 16.6 & 68 \\
\hline Saitama & 26.7 & 15.6 & 63 & Yamaguchi & 22.2 & 16.0 & 69 \\
\hline Chiba & 26.3 & 16.3 & 68 & Tokushima & 22.5 & 17.1 & 65 \\
\hline Tokyo & 24.8 & 16.7 & 59 & Kagawa & 23.6 & 16.9 & 65 \\
\hline Kanagawa & 26.0 & 16.3 & 64 & Ehime & 22.0 & 17.0 & 66 \\
\hline Niigata & 24.7 & 14.2 & 69 & Kochi & 23.6 & 17.6 & 68 \\
\hline
\end{tabular}


World Journal of Biology Pharmacy and Health Sciences, 2022, 09(01), 027-038

\begin{tabular}{|l|l|l|l|l|c|c|c|}
\hline Toyama & 23.5 & 14.6 & 78 & Fukuoka & 25.9 & 17.6 & 65 \\
\hline Ishikawa & 24.6 & 15.1 & 70 & Saga & 24.8 & 17.1 & 68 \\
\hline Fukui & 23.2 & 14.9 & 74 & Nagasaki & 23.2 & 17.7 & 67 \\
\hline Yamanashi & 25.4 & 15.3 & 62 & Kumamoto & 23.3 & 17.7 & 67 \\
\hline Nagano & 23.3 & 12.2 & 71 & Oita & 23.3 & 17.1 & 66 \\
\hline Gifu & 23.6 & 16.3 & 65 & Miyazaki & 23.5 & 17.9 & 71 \\
\hline Shizuoka & 24.6 & 17.0 & 69 & Kagoshima & 21.3 & 19.1 & 66 \\
\hline Aichi & 26.1 & 16.3 & 65 & Okinawa & 23.0 & 23.4 & 72 \\
\hline Mie & 23.4 & 16.4 & 68 & mean (SD) & $24.3(1.9)$ & $15.6(2.4)$ & $68(4)$ \\
\hline
\end{tabular}

"Average smoking rate of 2004 and 2007 surveys, "\#\# Average temperature from 2004 to 2007, "\#\# Average humidity from 2004 to 2007.

Table 8 Average concentration of photochemical oxidantss in the pre-school environment for first grade elementary school students from 2017 to 2019

\begin{tabular}{|c|c|c|c|c|c|c|c|}
\hline Prefecture & $\begin{array}{l}{ }^{\#} \text { Smoking } \\
\text { rate (\%) }\end{array}$ & $\begin{array}{c}{ }^{\# \#} \text { Ambient } \\
\text { temperature } \\
\left.\text { ( }{ }^{\circ} \mathrm{C}\right) \\
\end{array}$ & $\begin{array}{c}\text { \#\#\# Relative } \\
\text { humidity } \\
\text { (\%) }\end{array}$ & Prefecture & $\begin{array}{l}{ }^{\#} \text { Smoking } \\
\text { rate (\%) }\end{array}$ & $\begin{array}{c}{ }^{\# \#} \text { Ambient } \\
\text { temperature } \\
\left.\text { ( }{ }^{\circ} \mathrm{C}\right)\end{array}$ & $\begin{array}{c}{ }^{\# \# \#} \text { Relative } \\
\text { humidity } \\
\text { (\%) }\end{array}$ \\
\hline Hokkaido & 20.7 & 9.5 & 75 & Shiga & 18.5 & 15.3 & 73 \\
\hline Aomori & 26.1 & 10.9 & 75 & Kyoto & 19.3 & 16.5 & 66 \\
\hline Iwate & 24.9 & 11.0 & 70 & Osaka & 18.0 & 17.2 & 64 \\
\hline Miyagi & 23.0 & 13.2 & 73 & Hyogo & 21.1 & 17.2 & 64 \\
\hline Akita & 22.6 & 12.3 & 72 & Nara & 19.1 & 15.4 & 75 \\
\hline Yamagata & 21.9 & 12.3 & 69 & Wakayama & 17.0 & 17.2 & 67 \\
\hline Fukushima & 20.1 & 13.8 & 73 & Tottori & 19.3 & 15.5 & 74 \\
\hline Ibaraki & 23.8 & 14.5 & 67 & Shimane & 18.9 & 15.4 & 77 \\
\hline Tochigi & 22.5 & 14.6 & 61 & Okayama & 18.8 & 16.3 & 68 \\
\hline Gunma & 22.3 & 15.3 & 63 & Hiroshima & 19.2 & 16.7 & 66 \\
\hline Saitama & 22.6 & 15.7 & 65 & Yamaguchi & 19.3 & 15.9 & 75 \\
\hline Chiba & 21.9 & 16.6 & 65 & Tokushima & 19.4 & 17.0 & 69 \\
\hline Tokyo & 21.4 & 16.6 & 69 & Kagawa & 17.7 & 16.9 & 67 \\
\hline Kanagawa & 19.6 & 16.6 & 73 & Ehime & 18.4 & 16.9 & 69 \\
\hline Niigata & 19.9 & 14.1 & 73 & Kochi & 18.1 & 17.5 & 70 \\
\hline Toyama & 20.9 & 14.7 & 70 & Fukuoka & 20.6 & 17.6 & 70 \\
\hline Ishikawa & 19.6 & 15.2 & 74 & Saga & 22.0 & 17.2 & 72 \\
\hline Fukui & 20.5 & 15.1 & 62 & Nagasaki & 22.4 & 17.5 & 73 \\
\hline Yamanashi & 20.1 & 15.4 & 73 & Kumamoto & 20.6 & 17.3 & 73 \\
\hline Nagano & 21.9 & 12.5 & 65 & Oita & 20.9 & 16.9 & 71 \\
\hline Gifu & 19.8 & 16.5 & 68 & Miyazaki & 20.4 & 17.9 & 76 \\
\hline Shizuoka & 19.1 & 17.2 & 65 & Kagoshima & 20.7 & 19.0 & 73 \\
\hline Aichi & 20.8 & 16.5 & 67 & Okinawa & 18.6 & 23.5 & 73 \\
\hline Mie & 20.0 & 16.5 & 68 & mean (SD) & $20.5(1.9)$ & $15.8(2.3)$ & $70(4)$ \\
\hline
\end{tabular}




\subsection{Stepwise multiple linear regression analysis}

As shown in Table 9, stepwise multiple regression analysis with asthma rate as the objective variable, atopic dermatitis rate, smoking rate, photochemical oxidants, ambient temperature and relative humidity as explanatory variables, revealed that atopic dermatitis rate and smoking rate are significant independent variables.

Table 9 Stepwise multiple regression analysis with asthma rate as the objective variable, atopic dermatitis rate, smoking rate, photochemical oxidants, ambient temperature and relative humidity as explanatory variables

\begin{tabular}{|c|c|c|c|c|c|c|c|}
\hline & \multirow[t]{2}{*}{$\begin{array}{l}\text { Independent } \\
\text { variables }\end{array}$} & \multirow{2}{*}{$\begin{array}{c}\text { Estimated } \\
\text { regression } \\
\text { coefficient } \\
\text { B }\end{array}$} & \multicolumn{2}{|c|}{$\begin{array}{c}95 \% \\
\text { confidence } \\
\text { interval }\end{array}$} & \multirow{2}{*}{$\begin{array}{c}\text { Partial } \\
\text { regression } \\
\text { coefficient } \\
\beta\end{array}$} & \multirow[t]{2}{*}{$\begin{array}{c}\text { Cumulative } \\
\qquad \mathbf{R}^{2}\end{array}$} & \multirow[t]{2}{*}{$\begin{array}{c}P \text { - } \\
\text { value }\end{array}$} \\
\hline & & & $\begin{array}{l}\text { Lower } \\
\text { bound }\end{array}$ & $\begin{array}{l}\text { Upper } \\
\text { bound }\end{array}$ & & & \\
\hline \multirow{3}{*}{$\begin{array}{l}\text { First } \\
\text { investigation }\end{array}$} & Intercept & -3.780 & -6.571 & -0.990 & - & - & 0.008 \\
\hline & $\begin{array}{l}\text { Atopic } \\
\text { dermatitis } \\
\text { rate }\end{array}$ & 0.757 & 0.614 & 0.900 & 0.667 & 0.398 & 0.000 \\
\hline & Smoking rate & 0.204 & 0.095 & 0.314 & 0.236 & 0.452 & 0.000 \\
\hline \multirow{3}{*}{$\begin{array}{l}\text { Second } \\
\text { investigation }\end{array}$} & Intercept & -1.818 & -3.885 & 0.249 & - & - & 0.084 \\
\hline & $\begin{array}{l}\text { Atopic } \\
\text { dermatitis } \\
\text { rate }\end{array}$ & 0.643 & 0.496 & 0.790 & 0.574 & 0.351 & 0.000 \\
\hline & Smoking rate & 0.160 & 0.060 & 0.260 & 0.211 & 0.395 & 0.002 \\
\hline
\end{tabular}

As shown in Table 10, in the first investigation of stepwise multiple regression analysis with atopic dermatitis rate among first grade elementary school students as the objective variable, smoking rate, photochemical oxidants, ambient temperature and relative humidity as explanatory variables, photochemical oxidants were significant independent variable. Ambient temperature and photochemical oxidants were significant independent variables in the second investigation.

Table 10 Stepwise multiple regression analysis with atopic dermatitis rate as the objective variable, smoking rate, photochemical oxidants, ambient temperature and relative humidity as explanatory variables

\begin{tabular}{|c|c|c|c|c|c|c|c|}
\hline & \multirow[t]{2}{*}{$\begin{array}{c}\text { Independent } \\
\text { variables }\end{array}$} & \multirow{2}{*}{$\begin{array}{c}\text { Estimated } \\
\text { regression } \\
\text { coefficient } \\
\text { B }\end{array}$} & \multicolumn{2}{|c|}{$\begin{array}{c}\text { 95\% confidence } \\
\text { interval }\end{array}$} & \multirow{2}{*}{$\begin{array}{c}\text { Partial } \\
\text { regression } \\
\text { coefficient } \\
\beta\end{array}$} & \multirow[t]{2}{*}{$\begin{array}{c}\text { Cumulative } \\
\mathbf{R}^{2}\end{array}$} & \multirow[t]{2}{*}{$\begin{array}{c}P \text { - } \\
\text { value }\end{array}$} \\
\hline & & & $\begin{array}{l}\text { Lower } \\
\text { bound }\end{array}$ & $\begin{array}{l}\text { Upper } \\
\text { bound }\end{array}$ & & & \\
\hline \multirow{2}{*}{$\begin{array}{l}\text { First } \\
\text { investigation }\end{array}$} & Intercept & -1.019 & 0.013 & 0.054 & - & - & 0.478 \\
\hline & $\begin{array}{l}\text { Photochemical } \\
\text { oxidants }\end{array}$ & 143.527 & 54.433 & 232.621 & 0.261 & 0.068 & 0.002 \\
\hline \multirow{3}{*}{$\begin{array}{l}\text { Second } \\
\text { investigation }\end{array}$} & Intercept & 1.549 & -2.005 & 5.103 & - & - & 0.390 \\
\hline & $\begin{array}{l}\text { Ambient } \\
\text { Temperature }\end{array}$ & -0.146 & -0.233 & -0.059 & -0.267 & 0.092 & 0.001 \\
\hline & $\begin{array}{l}\text { Photochemical } \\
\text { oxidants }\end{array}$ & 118.685 & 28.625 & 208.744 & 0.210 & 0.135 & 0.010 \\
\hline
\end{tabular}

Figure 1 shows the relationship between photochemical oxidants and atopic dermatitis based on the results of the first investigation. 


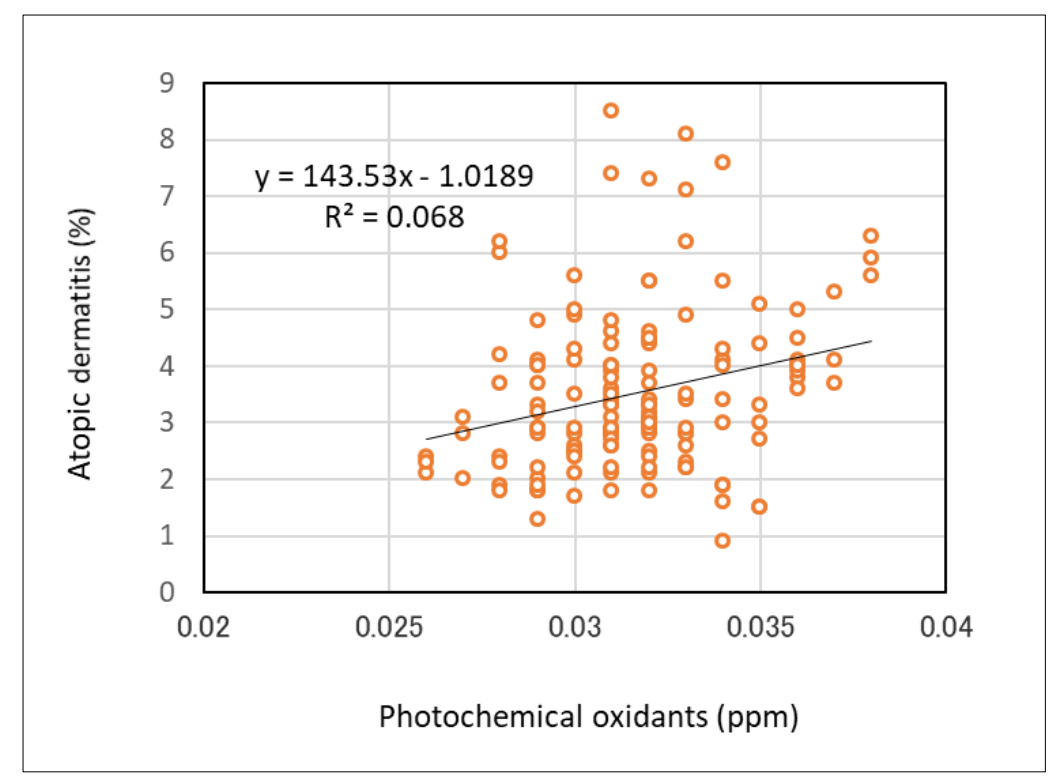

Figure 1 Relationship between photochemical oxidants and atopic dermatitis based on the results of the first investigation

\section{Discussion}

Air pollutants and climatic conditions are potential factors in the development of childhood asthma and atopic dermatitis [8,9]. Asthma is usually classified as atopic and non-atopic [10]. Atopy is associated with allergens that cause allergic reactions [11]. The results shown in Table 5 suggest that the tobacco smoke is a risk factor for non-atopic asthma rather than atopic asthma [12-17]. Tobacco smoke is an irritant that irritates the airways and can exacerbate asthma and cause those asthma symptoms [18-20]. Photochemical oxidants and ambient temperature were significant independent variables for atopic dermatitis. The relationship between the photochemical oxidant concentration in Figure 1 and the incidence of atopic dermatitis may suggest a dose-response relationship. However, there is a limitation that the results of regression analysis cannot prove a causal relationship.

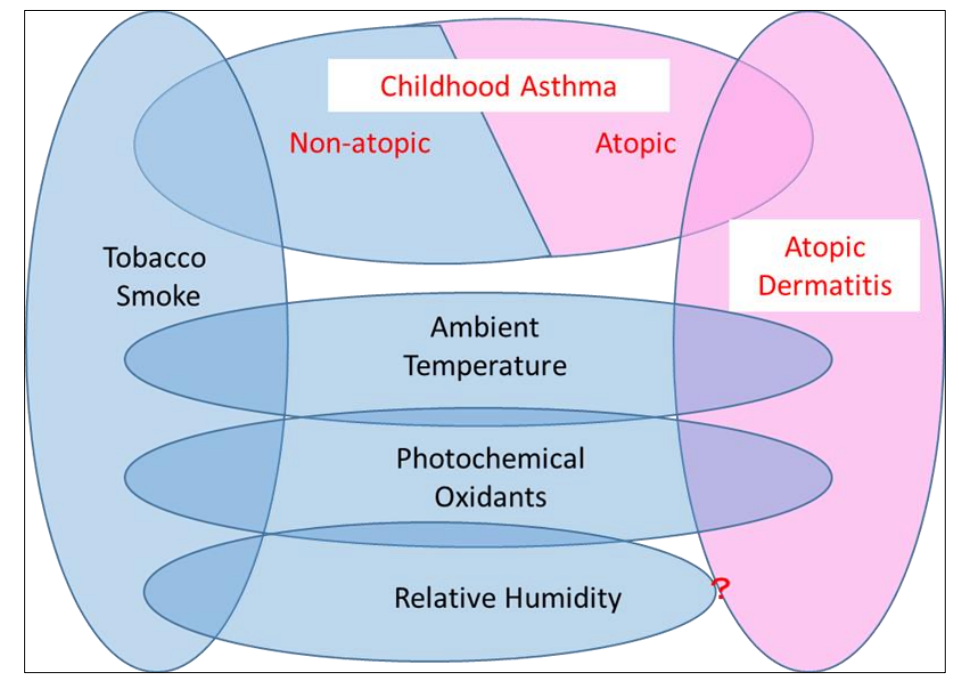

Figure 2 Relationship between childhood asthma, atopic dermatitis and environmental factors

Photochemical oxidants are strong oxidative stressors that produce reactive oxygen species (ROS), such as superoxide $\left(\mathrm{O}_{2}-\right)$ and hydrogen peroxide $\left(\mathrm{H}_{2} \mathrm{O}_{2}\right)$. ROS causes skin barrier dysfunction and inflammation that develops atopic dermatitis [21-24]. Cold temperatures can cause allergic reactions such as cold urticarial, leading to a reproducible and significant increase in histamine-induced itching [25-27]. Itching is a major symptom of many allergic or inflammatory 
skin disorders. In addition, low humidity and low temperatures lead to a general reduction in skin barrier function, making them more susceptible to mechanical stress [28].

When the results obtained in this study and the research results reported by the author [29, 30] are combined, the relationship between childhood asthma and atopic dermatitis and environmental factors such as tobacco smoke, temperature, and humidity is as follows (Figure 2).

\section{Conclusion}

Present study suggests that preschool indoor and outdoor conditions such as environmental tobacco smoke, photochemical oxidants, and ambient temperature may be associated with the development of childhood asthma and atopic dermatitis.

\section{Compliance with ethical standards}

\section{Acknowledgments}

The author appreciates the help of colleagues in the laboratory.

\section{Disclosure of conflict of interest}

There is no conflict of interest in this work.

\section{References}

[1] Elina Toskala, David W Kennedy, Asthma risk factors. International Forum of Allergy \& Rhinology, Vol. 5, No. S1, September 2015 S16

[2] D Solé, IC Camelo-Nunes,1 GF Wandalsen, AC Pastorino, CMA Jacob, C Gonzalez, NF Wandalsen, NA Rosário Filho, GB Fischer, CK Naspitz. Prevalence of Symptoms of Asthma, Rhinitis, and Atopic Eczema in Brazilian Adolescents Related to Exposure to Gaseous Air Pollutants and Socioeconomic Status. J Investig Allergol Clin Immunol. 2007; 17(1): 6-13.

[3] Ki Lee Milligan, Elizabeth Matsui, Hemant Sharma, Asthma in Urban Children: Epidemiology, Environmental Risk Factors, and the Public Health Domain. Curr Allergy Asthma Rep. 2016; Apr; 16(4): 33.

[4] A Vanker, R P Gie, H J Zar. The association between environmental tobacco smoke exposure and childhood respiratory disease: a review. Expert Rev Respir Med. 2017; 11(8): 661-673.

[5] Eleni Drakaki, Clio Dessinioti, Christina V. Antoniou, Air pollution and the skin. Front Environ. Sci. 15 May 2014; 00011.

[6] Dotterud LK, Kvammen B, Bolle R, Falk ES, A survey of atopic diseases among school children in Sør-Varanger community. Possible effects of subarctic climate and industrial pollution from Russia. Acta Derm Venereol (Stockh). 1994; 74: 124-128.

[7] JSC English, RS Dawe, J Ferguson. Environmental effects and skin disease. Br Med Bull. 68: 129-142.

[8] Yabin Hu, Zhiwei Xu, Fan Jiang, Shenghui Li, Shijian Liu, Meiqin Wu, Chonghuai Yan, Jianguo Tan, Guangjun Yu, Yi $\mathrm{Hu}$, Yong Yin, Shilu Tong. Relative impact of meteorological factors and air pollutants on childhood allergic diseases in Shanghai, China. Sci Total Environ. 1 Mar 2020; 706: 135975.

[9] Juanjuan Zhang, Jihong Dai Li Yan, Wenlong Fu, Jing Yi, Yuzhi Chen, Chuanhe Liu, Dongqun Xu, Qiang Wang. Air Pollutants, Climate, and the Prevalence of Pediatric Asthma in Urban Areas of China. BioMed Research International. 2016; 2935163: 8.

[10] CS Court, DG Cook, DP Strachan. Comparative epidemiology of atopic and non-atopic wheeze and diagnosed asthma in a national sample of English adults. Thorax. 2002; 57(11): 951-957.

[11] Simon F Thomsen. Epidemiology and natural history of atopic diseases. Eur Clin Respir J. 2015; 2: 10.3402/ecrj.v2.24642. 
[12] E Rönmark, E Jönsson, T Platts-Mills, B Lundbäck. Different pattern of risk factors for atopic and nonatopic asthma among children--report from the Obstructive Lung Disease in Northern Sweden Study, Allergy. 1999; 54(9): 926-935.

[13] Juha Pekkanen, Jussi Lampi, Jon Genuneit, Anna-Liisa Hartikainen, Marjo-Riitta Järvelin. Analyzing atopic and non-atopic asthma. Eur J Epidemiol. 2012; 27(4): 281-286.

[14] Donna C Rennie, Chandima P Karunanayake, Josh A Lawson, Shelley Kirychuk, Kathleen McMullin, Sylvia Abonyi, Jeremy Seeseequasis, Judith MacDonald, James A Dosman, Punam Pahwa, Domestic Risk Factors for Atopic and non-atopic asthma in first nations children living in Saskatchewan, Canada. Children (Basel). 2020; 7 (5): 38.

[15] Ana Lucia Moncayo, Maritza Vaca, Gisela Oviedo, Silvia Erazo, Isabel Quinzo, Rosemeire L Fiaccone, Martha E Chico, Mauricio L Barreto, Philip J Cooper, Risk factors for atopic and non-atopic asthma in a rural area of Ecuador. Thorax. 2010; 65(5): 409-416.

[16] Hussain Booalayan, Mosa Abdualrasool, Saad Al-Shanfari, Abdulwahab Boujarwa, Abdullah Al-Mukaimi, Omar Alkandery, Saeed Akhtar. Exposure to environmental tobacco smoke and prevalence of asthma among adolescents in a middle eastern country. BMC Public Health. 2010; 20: 1210.

[17] DP Strachan, DG Cook. Health effects of passive smoking. 6. Parental smoking and childhood asthma: longitudinal and case-control studies. Thorax. 1998; 53(3): 204-212.

[18] Emil Bogen. Irritant Factors in Tobacco Smoke. California and Western Medicine. 1936; 45(4): $342-346$.

[19] RJ Shephard. Respiratory irritation from environmental tobacco smoke. Arch Environ Health. 1992; 47(2): 123130 .

[20] Gilmour MI, Jaakkola MS, London SJ, Nel AE, Rogers CA. How exposure to environmental tobacco smoke, outdoor air pollutants, and increased pollen burdens influences the incidence of asthma. Environ Health Perspect. 2006; 114(4): 627-633.

[21] Sukriti Sharma, Amarjit S Naura. Potential of phytochemicals as immune-regulatory compounds in atopic diseases: A review. Biochem Pharmacol. 2020; 173: 113790.

[22] Hongxiu Ji, Xiao-Kang Li. Oxidative Stress in Atopic Dermatitis. Oxidative Medicine and Cellular Longevity. 2016, 2721469: 8 .

[23] Lucrezia Bertino, Fabrizio Guarneri, Serafinella Patrizia Cannavò, Marco Casciaro, Giovanni Pioggia and Sebastiano Gangemi, Oxidative Stress and Atopic Dermatitis. Antioxidants. 2020; 9(3): 196.

[24] Yoshimichi Okayama, Oxidative stress in allergic and inflammatory skin diseases. Curr Drug Targets Inflamm Allergy. 2005; 4(4): 517-519.

[25] Florian Pfab, Michael Valet, Till Sprenger, Thomas R Toelle, Georgios I Athanasiadis, Heidrun Behrendt, Johannes Ring, Ulf Darsow, Short-term alternating temperature enhances histamine-induced itch: a biphasic stimulus model. J Invest Dermatol. 2006; 126(12): 2673-2678.

[26] F Pfab, M Valet, T Sprenger, J Huss-Marp, G I Athanasiadis, HJ Baurecht, A Konstantinow, C Zimmer, H Behrendt, J Ring, T R Tölle, U Darsow, Temperature modulated histamine-itch in lesional and nonlesional skin in atopic eczema - a combined psychophysical and neuroimaging study. Allergy. 2010; 65(1): 84-94.

[27] JSC English, RS Dawe, J Ferguson. Environmental effects and skin disease. Br Med Bull. 68: 129-142.

[28] KA Engebretsen, JD Johansen, S Kezic, A Linneberg, JP Thyssen. The effect of environmental humidity and temperature on skin barrier function and dermatitis. J Eur Acad Dermatol Venereol. 2016; 30(2): 223-249.

[29] S Suna, Pollutants and climatic conditions related to the smoking rate. World Journal of Biology Pharmacy and Health Sciences. 2021; 8(2): 34-41.

[30] S Suna. Pollutants and climatic conditions related to the formation of photochemical oxidants. World Journal of Biology Pharmacy and Health Sciences. 2021; 5(2): 1-5. 